Elsevier Editorial System(tm) for Placenta Manuscript Draft

Manuscript Number: PL-15-00058R3

Title: Ultrasound And Endocrinological Markers of First Trimester Placentation And Subsequent Fetal Size

Article Type: Original Article

Keywords: Placental volume; birth weight; placental hormones; large for gestational age; small for gestational age

Corresponding Author: Dr. Anna Louise David, MB ChB, MRCOG, PhD

Corresponding Author's Institution: University College London

First Author: Anna Louise David, MB ChB, MRCOG, PhD

Order of Authors: Anna Louise David, MB ChB, MRCOG, PhD; Eric R Jauniaux, PhD FRCOG

Abstract: Introduction: To study the relationship between 2-dimensional placental ultrasound measurements and maternal serum (MS) levels of biomarkers of placentation and in pregnancies presenting with an isolated abnormally high or low birthweight at term, without evidence of placental insufficiency.

Method: We performed a population based cohort study of 306 pregnancies delivered at term including 30 presenting with large-for-gestational age (LGA, birthweight > 90th centile) and 17 small-for-gestational age (SGA; birthweight < 10th centile). Antenatal measurements included placental thickness and 2D-volume and MS levels of pregnancy-associated plasma protein A (PAPP-A) and free-beta human chorionic gonadotrophin (fßhCG) at 11 - 13+6 weeks of gestation and mid-trimester MS $\alpha$-fetoprotein (AFP), unconjugated estriol (uE3) and inhibin A levels.

Results: In the subgroup with a normal birthweight (10th-90th centile), there was a significant positive correlation between birthweight and the basal plate surface area $(\mathrm{p}<0.001)$ and $2 \mathrm{D}$ placental volume $(\mathrm{p}<0.01)$. In the LGA subgroup, MS PAPP-A was significantly $(\mathrm{p}<0.05)$ higher than in normal controls and there was a significant $(\mathrm{p}<0.01)$ positive correlation with birthweight. There was no significant difference for any of the ultrasound and biomarkers parameters between SGA and the normally grown controls.

Discussion: In uncomplicated singleton pregnancies with a normal

birthweight, 2D measurements of placentation are related with fetal size but are not related to subsequent excessive or slow fetal growth. LGA at birth is associated with increased MS PAPP-A at 11-14 weeks of gestation supporting the association between PAPP-A synthesis and early placental growth and development. 
Dear Graham

As requested, I have added in the Supplementary Figures 1 and 2 (Scatter plot illustrating the relationship between first trimester placental surface area or 2D volume and birthweight for the whole cohort), Eric has added the statistical data for these results into the results section on page 10 . He also corrected 2 typos and added 2 decimals in all the F numbers for consistency.

Yours sincerely

Anna David 
${ }^{*}$ Conflict of Interest Statement

Click here to download Conflict of Interest Statement: Conflict of Interest Statement.docx

Conflict of Interest Statement

The authors state that no conflict of interest exists. 


\section{Ultrasound And Endocrinological Markers Of First Trimester Placentation And Subsequent Fetal Size}

A.L. DAVID, E. JAUNIAUX

Research Department of Maternal Fetal Medicine, Institute for Women's Health, University College London, 86-96 Chenies Mews, London WC1E $6 \mathrm{HX}, \mathrm{UK}$.

Correspondence address:

Dr Anna David,

Research Department of Maternal Fetal Medicine,

Institute for Women's Health

University College London,

86-96 Chenies Mews,

London WC1E 6HX, UK.

Telephone numbers:

$+44 / 207 / 6796059$

Fax: $+44 / 207 / 3837429$

a.david@ucl.ac.uk 


\section{ABSTRACT (243 words)}

Introduction: To study the relationship between 2-dimensional placental ultrasound measurements and maternal serum (MS) levels of biomarkers of placentation and in pregnancies presenting with an isolated abnormally high or low birthweight at term, without evidence of placental insufficiency.

Method: We performed a population based cohort study of 306 pregnancies delivered at term including 30 presenting with large-for-gestational age (LGA, birthweight $>90^{\text {th }}$ centile) and 17 small-for-gestational age (SGA; birthweight $<10^{\text {th }}$ centile). Antenatal measurements included placental thickness and 2Dvolume and MS levels of pregnancy-associated plasma protein A (PAPP-A) and free-beta human chorionic gonadotrophin ( $f(\beta h C G)$ at $11-13^{+6}$ weeks of gestation and mid-trimester MS a-fetoprotein (AFP), unconjugated estriol (uE3) and inhibin A levels.

Results: In the subgroup with a normal birthweight $\left(10^{\text {th }}-90^{\text {th }}\right.$ centile), there was a significant positive correlation between birthweight and the basal plate surface area $(p<0.001)$ and 2D placental volume $(p<0.01)$. In the LGA subgroup, MS PAPP-A was significantly $(p<0.05)$ higher than in normal controls and there was a significant $(p<0.01)$ positive correlation with birthweight. There was no significant difference for any of the ultrasound and biomarkers parameters between SGA and the normally grown controls.

Discussion: In uncomplicated singleton pregnancies with a normal birthweight, 2D measurements of placentation are related with fetal size but are not related to subsequent excessive or slow fetal growth. LGA at birth is 
associated with increased MS PAPP-A at 11-14 weeks of gestation supporting the association between PAPP-A synthesis and early placental growth and development.

Keywords: Placental volume, birth weight, placental hormones, large for gestational age, small for gestational age 
Highlights

- In a population based cohort study of 306 uncomplicated singleton pregnancies delivered at term, 2D placental measurements correlate with fetal size in the total study group and To study the relationship between $2 D$ placental measurements, maternal placental biomarkers and fetal growth complications

- in uncomplicated singleton pregnancies with a normal birthweight 2D placental measurements correlate with fetal size

- Placental basal plate surface area and 2D placental volume do not predict large or small fetal size and these measurements cannot be used to predict birthweight in individual cases..

- Large (LGA) and small for gestational age (SGA) at birth are not associated with 2D ultrasound measurements of the placenta

- LGA but not SGA is associated with raised first trimester maternal serum pregnancy-associated plasma proteinFirst trimester maternal serum pregnancy-associated plasma protein concentration is higher in LGA pregnancies than compared with normal and AGA pregnancies. 


\section{Ultrasound And Endocrinological Markers Of 2 First Trimester Placentation And Subsequent 3 Fetal Size}

4

5 A.L. DAVID, E. JAUNIAUX

6

7 Research Department of Maternal Fetal Medicine, Institute for Women's

8 Health, University College London, 86-96 Chenies Mews, London WC1E $96 \mathrm{HX}, \mathrm{UK}$.

14 Correspondence address:

15 Dr Anna David,

16 Research Department of Maternal Fetal Medicine,

17 Institute for Women's Health

18 University College London,

19 86-96 Chenies Mews,

20 London WC1E 6HX, UK.

21 Telephone numbers:

$22+44 / 207 / 6796059$

23 Fax: $+44 / 207 / 3837429$

24 a.david@ucl.ac.uk 


\section{ABSTRACT (243 words)}

29 Introduction: To study the relationship between 2-dimensional placental

30 ultrasound measurements and maternal serum (MS) levels of biomarkers of

31 placentation and in pregnancies presenting with an isolated abnormally high

32 or low birthweight at term, without evidence of placental insufficiency.

33 Method: We performed a population based cohort study of 306 pregnancies

34 delivered at term including 30 presenting with large-for-gestational age (LGA,

35 birthweight $>90^{\text {th }}$ centile) and 17 small-for-gestational age (SGA; birthweight

$36<10^{\text {th }}$ centile). Antenatal measurements included placental thickness and 2D-

37 volume and MS levels of pregnancy-associated plasma protein A (PAPP-A)

38 and free-beta human chorionic gonadotrophin ( $\mathrm{f} \beta \mathrm{hCG}$ ) at $11-13^{+6}$ weeks of

39 gestation and mid-trimester MS a-fetoprotein (AFP), unconjugated estriol

40 (UE3) and inhibin A levels.

41 Results: In the subgroup with a normal birthweight $\left(10^{\text {th }}-90^{\text {th }}\right.$ centile), there

42 was a significant positive correlation between birthweight and the basal plate

43 surface area $(p<0.001)$ and $2 D$ placental volume $(p<0.01)$. In the LGA

44 subgroup, MS PAPP-A was significantly $(p<0.05)$ higher than in normal

45 controls and there was a significant $(p<0.01)$ positive correlation with

46 birthweight. There was no significant difference for any of the ultrasound and

47 biomarkers parameters between SGA and the normally grown controls.

Discussion: In uncomplicated singleton pregnancies with a normal

49 birthweight, 2D measurements of placentation are related with fetal size but

50 are not related to subsequent excessive or slow fetal growth. LGA at birth is 
51 associated with increased MS PAPP-A at 11-14 weeks of gestation

52 supporting the association between PAPP-A synthesis and early placental

53 growth and development.

54 Keywords: Placental volume, birth weight, placental hormones, large for

55 gestational age, small for gestational age

56 


\section{Introduction}

Major changes take place in the uterine environment between the first trimester and the rest of pregnancy [1]. These changes are characterised by the establishment of the intervillous circulation, a switch from histiotrophic to haemotrophic fetal nutrition and the remodelling of the primitive placenta [2]. In early pregnancy, the oxygen concentration within the chorionic sac is maintained at a low value to protect the primitive placenta and the developing fetus from the teratogenic effect of oxygen free radicals $[1,2]$. The onset of the maternal arterial circulations at the end of the first trimester leads to a threefold rise in the intraplacental oxygen concentration $[1,3]$. The haemodynamics of the utero-placental circulation and distribution of oxygen around and inside the placenta play an important role in shaping the topography of the villous tree of the definitive placenta and the formation of the free placental membranes [1-3].

Fetal size is dependent on nutrient availability, which in turn is related to villous development and the capacity of the villous trophoblast to transport these nutrients [4]. Sufficient dilation of the utero-placental circulation together with rapid villous angiogenesis are the key factors necessary for adequate placental development and function, and subsequent fetal growth. Placental and fetal development is related and placental growth kinetics are important features predicting post-natal health and in particular cardiovascular adaptations in childhood, even when fetal size is in the normal range [5]. At birth, there is a correlation between fetal and placental weight and the ratio of these weights i.e. the feto-placental weight ratio (FPR) gives retrospectively 
81 an indication of the appropriateness of fetal growth during pregnancy and

82 estimates the potential risks for chronic diseases in later life [6].

The placenta actively produces a large number of hormones that serve to regulate and balance maternal and fetal physiology and in particular fetal growth via the production and metabolism of growth-regulating hormones [7]. The expression of numerous signaling molecules is altered in the placentas from pregnancies affected by the fetal growth complications of fetal growth restriction and macrosomia. Many maternal serum (MS) biomarkers have been proposed for the detection of abnormal fetal growth but when used alone in the first trimester they have a limited role as screening tests. For example, pregnancy-associated plasma protein-A (PAPP-A) during the first trimester cannot be used alone as a marker of excessive fetal growth, large [8] or of small-for-gestational age (SGA) [9,11]. Similarly new biomarkers such as adiponectin, a hormone that regulates glucose levels and fatty acid oxidation, have been recently tested in the first trimester to predict fetal size later in pregnancy with variable results $[12,13]$.

For decades, ultrasound remains the best method for the screening diagnosis, characterization and follow-up of abnormal fetal growth. The advent of Doppler ultrasound has enabled the study of the development of the uterine and umbilical circulations in utero from early in normal pregnancy [14]. Further studies have shown an association between uterine circulation, abnormal placental features including size, abnormal level of biomarkers in MS and abnormal fetal growth [15,16]. More recently 3-dimensional (3D) ultrasound has enabled the study of placental volume in utero throughout pregnancy. These studies have shown an association between placental 
106 volume at 11-13 weeks and birth weight in pregnancies with a normal and 107 complicated outcome [17-22]. The combination of the assessment of placental size and shape with 109 3D ultrasound and MS biomarkers can serve as the foundation upon which to 110 build a multivariate model for the early prediction of abnormal fetal size. The

111 broad variability in placental volume 3D measurements and current time-

112 consuming approach compared to 2D ultrasound has made its clinical use

113 difficult [23]. In this study, we investigated the relationship between first

114 trimester (11 - $13^{+6}$ weeks of gestation) 2D ultrasound measurements of 115 placentation and MS levels of commonly measured placental proteins PAPP-

116 A and free-beta human chorionic gonadotrophin ( $f \beta h C G)$, and mid-trimester $117\left(15-22^{+0}\right.$ weeks of gestation) inhibin and subsequent fetal size.

\section{Patients and methods}

120 A group of 476 women booked for antenatal care at University College

121 London Hospital (UCLH) were recruited prospectively over a 40-month period.

122 All pregnant women at UCLH are offered a screening test for Trisomy 21

123 which combines nuchal translucency (NT) measurement and MS levels of

124 PAPP-A and $\mathrm{f} \beta \mathrm{hCG}$ at $11-13^{+6}$ weeks (combined test) and/or of AFP, uE3

125 and inhibin-A at $15-22^{+0}$ weeks (integrated test). All women were recruited at

126 the time of the NT ultrasound examination between $11^{+0}$ and $13^{+6}$ weeks of 127 gestation (77-97 gestational days) as determined either by menstrual age, or 

medical condition, multiple pregnancy and abnormal pregnancy outcome

144 including, late miscarriage, intrauterine death, stillbirth, preterm $(<37$ weeks of

when there was a discrepancy of more than 5 days by the ultrasound measurement of the fetal crown-rump length $(C R L)$.

Demographic data including maternal age, ethnicity, parity, cigarette smoke exposure, age and body mass index (BMI) were collected from questionnaires completed at the time of the first appointment. All women underwent routine screening for gestational diabetes with a random glucose at booking and at 28 weeks of gestation. In addition women with specific risk factors (previous LGA, family history of diabetes) were offered a formal 2 hour glucose tolerance test at 28 weeks. Pregnancy outcome information was collected from the medical case notes and hospital electronic patient records.

The birthweight percentile was calculated using the customized gestational age related optimal growth chart (GROW) (www.gestation.net), which takes into account ethnicity, maternal height, weight, age and fetal birthweight and gender.

Women diagnosed with a first trimester miscarriage, fetal abnormality, gestation) or post-term delivery ( $\geq 41+6$ weeks of gestation), smokers or lost to follow-up were excluded from the analysis. Placental pathology results were available for cases of SGA and IUGR identified at birth.

\subsection{Ultrasound examination}

All examinations were performed using a 3.5-5 MHz ultrasound probe (Voluson 730 and E8 Expert, GE, USA). In all cases, the fetal NT, CRL and basic anatomy were obtained. The placental basal plate dimensions and 
152 thickness were measured by viewing the whole placenta as previously

153 described $[25,26]$. Three measurements of each parameter were taken and

154 they were subsequently averaged for analysis. In brief the longest sagittal and

155 transverse diameters of the placental basal plate were measured at the level

156 of the utero-placental interface and the placental thickness was measured

157 underneath the cord insertion. The basal plate surface area of the placenta

158 was estimated using the following formula: Sagittal length $x$ transverse length

$159 x \pi / 4$. The placental volume was calculated as being the equation for half the

160 volume of an ellipsoid: $1 / 2$ (sagittal length $x$ transverse length $x$ thickness $x$

$1614 / 3 \pi)$, as previously described [24]. The placental measurements were

162 entered into the prospective study database at the time of the ultrasound

163 scan. Four sonographers were trained by the study authors prior to the study

164 commencement so as to perform the placental ultrasound measurements in a 165 reproducible and systematic way.

\subsection{Bioassays}

MS PAPP-A and $f \beta h C G$ levels were measured using the AutoDELFIA PAPP-

168 A, time-resolved fluoro-immunoassay (PerkinElmer, Turku, Finland). Inhibin A

169 MS levels were measured using a commercial ELISA. The measured proteins

170 were converted to multiple of the median (MoM) for a pregnancy of the same

171 gestational age and adjusted for maternal age and ethnicity.

\section{$172 \quad 2.3$ Study group and statistical analysis}

The study group included 306 non-smoking women with a spontaneous

174 singleton pregnancy delivering after an uncomplicated pregnancy between

$17537^{+1}$ weeks (259 days of pregnancy) and $41^{+6}$ weeks (286 days of pregnancy). 
176 The study group was divided into 3 subgroups according to the neonatal 177 weight percentile at birth adjusted for neonatal gender [24]. Cases in the 178 large-for-gestatonal age (LGA, birth weight $>90^{\text {th }}$ centile) and SGA (birth 179 weight $<10^{\text {th }}$ centile with no evidence of fetal growth restriction, as defined as 180 abnormal umbilical artery and uterine artery Doppler or abnormal 181 cerebroplacental ratio) subgroup were individually matched for maternal BMI, 182 parity and for the CRL with three cases from the normal $\left(10-90^{\text {th }}\right.$ centile) 183 subgroup. The study was approved by the Joint UCL/UCLH Committees on 184 the Ethics of Human Research (Reference Number: 05/Q0505/82). All women 185 received information about the study and written consent was obtained prior 186 to the ultrasound examination. The data were analyzed using the StatGraphic data analysis and

188 statistical software package (Station, TX). Standard Kurtosis analysis 189 indicated that some values were not normally distributed and are therefore 190 presented as median and interquartile range (IQR). The median values of the 191 different variable investigated in the macrosomic and SGA subgroups and 192 controls were compared using a Mann-Whitney (Wilcoxon) W test. Individual 193 correlations between the different ultrasound, endocrinologic and clinical 194 variables were calculated by the least square method and their slopes tested 195 for significance by the $F$ ratio test. A p value of $<0.05$ was considered 196 significant.

\section{Results}

198 There were $259(84.6 \%)$ pregnancies with a birthweight between $10^{\text {th }}$ and $90^{\text {th }}$ 199 centile (normal), $30(9.8 \%)$ with a birthweight $>90^{\text {th }}$ centile (LGA) and 17 $200 \quad(5.6 \%)$ with a birthweight below the $10^{\text {th }}$ centile (SGA). 


\begin{tabular}{l|l}
201 & The regression analysis between birthweight and the ultrasound and \\
202 & $\begin{array}{l}\text { endocrinologic parameters in the entire study group indicated a significant } \\
\text { positive correlation with placental basal surface area }(F=11.03 ; r=0.41:\end{array}$ \\
203 & $\begin{array}{l}\text { P<0.005) and 2D volume }(F=7.60 ; r=0.29, p<0.01),(\text { Supplementary Figures } 1 \\
205\end{array}$ \\
206 & $\begin{array}{l}\text { and 2) and MS PAPP-A concentration }(F=5.10 ; r=0.14, p<0.05), \text { but no such } \\
\text { relationship existed for MS } \mathrm{f} \beta \mathrm{hCG} .\end{array}$
\end{tabular}

\subsection{Normal birth weight}

At the time of the ultrasound examination, the median $C R L$ was $62.3 \mathrm{~mm}$ (IQR 56.1;70). Using the NIH BMI calculator (www.nhlbi.nih.gov/ guidelines/obesity/

$210 \mathrm{BMI} /$ bmicalc.htm), 12 women were classified at the antenatal booking

211 appointment (8-10 weeks of gestation) as underweight (BMI < 18.5), 181 as

212 normal weight (BMI 18.5-24.9), 52 as overweight (BMI 25-29.9 and 14 as

213 obese $(\geq 30)$

Table 1 displays and compares the clinical, ultrasound and

215 endocrinological data of women with a $\mathrm{BMI}<25$ with those with a $\mathrm{BMI} \geq 25$.

216 The MS level of $f \beta h C G$ at $11-13^{+6}$ weeks was significantly $(p<0.05)$ higher in

217 women with a $\mathrm{BMI} \geq 25$. There was no significant difference for the other

218 parameters between the two subgroups.

There was a significant positive correlation between the basal plate

220 surface area $(F=10.3 \underline{0} ; r=0.20, p<0.001)$ and placental volume $(F=7.6 \underline{0}$;

$221 r=\underline{0.17}, \mathrm{p}<0.01)$ at $11-13^{+6}$ weeks and subsequent fetal size. There was no

222 significant correlation between MS $f \beta h C G$ or PAPP-A at $11-13^{+6}$ weeks and

223 mid-trimester AFP, UE3 and inhibin MS levels and fetal size. 


\subsection{LGA}

225 The median birthweight was 4128g in the LGA subgroup (IQR 4000;4350)

226 compared to $3289 \mathrm{~g}$ in the AGA subgroup (IQR $3121 ; 3487, \mathrm{p}<0.001$ ). The

227 concentration of MS PAPP-A at $11-13^{+6}$ weeks was significantly $(p<0.05)$

228 higher in the serum of the women delivering an LGA neonate than in controls

229 with a normal birthweight at term (Table 2). There was no significant

230 difference for the ultrasound parameters in this subgroup. There was a

231 significant positive correlation $(F=8.03 ; r=0.20, p<0.01)$ between MS PAPP-A

232 concentration and increased birthweight_at term_(Figure 1), but no such

233 relationship existed for $\mathrm{MS} \mathrm{f} \beta \mathrm{hCG}$ AFP, uE3 and inhibin.

$234 \quad 3.4$ SGA

235 The median birthweight was $2850 \mathrm{~g}$ in the SGA subgroup (IQR 2800;2922)

236 compared to $3462 \mathrm{~g}$ in the AGA control subgroup (IQR 3206;3632, $\mathrm{p}<0.001$ ).

237 There was no significant difference for the ultrasound and endocrinologic

238 parameters between SGA and control pregnancies (Table 3). There was no

239 significant correlation between MS- $\underline{\text { hormonal levels }}$ at $11-13^{+6}$ weeks and mid-

240 trimester and SGA. 
243 Our results indicate a relationship between 2D measurements of the

244 placentation area and volume, MS PAPP-A at $11-13^{+6}$ weeks of gestation and

245 birthweight in uncomplicated singleton pregnancies that deliver at term. In

246 otherwise uncomplicated pregnancies, presenting with LGA or SGA at term,

$2472 \mathrm{D}$ first-trimester ultrasound measurements of placentation are not related to

248 abnormal fetal size and only MS PAPP-A are related to subsequent excessive 249 fetal size.

There are well-documented associations between the levels of first and

251 second trimester MS biomarkers and abnormal placental development in 252 pregnancies subsequently complicated by pre-eclampsia and intrauterine 253 growth restriction (IUGR). A longitudinal study has shown that in cases of 254 IUGR, with or without accompanying pre-eclampsia, the placenta is already 255 smaller at 12-18 weeks of gestation on ultrasound than in healthy controls 256 [26]. In the most severe cases of deficient arterial conversion the intervillous 257 circulation is abnormal from the beginning of the second trimester, resulting in 258 pronounced ultrasonographic changes in placental texture $[14,27,28]$, that are 259 associated with IUGR, pre-eclampsia and high MS alpha-fetoprotein levels at $26018-28$ weeks [14]. In these pregnancies, there is a narrow implantation basis

261 or basal plate diameter, increased thickness and patchy decrease in placental 262 echogenicity secondary to the fetal plate being pushed up by jet-like blood 263 streams from the spiral arteries [14]. We have previously shown that in 264 pregnancies subsequently complicated by pre-eclampsia, the levels of MS 265 PAPP-A are lower at the end of the first trimester and the basal plate surface 266 area is smaller [24] reflecting indirectly the development of the definitive 
placenta $[24,25]$. These findings support the concept that placental-related complications of the second-half of gestation have their pathophysiological origin in abnormal placentation and utero-placental development during the

270 first trimester of pregnancy [3].

In the present study, we have excluded pregnancies with an existing or

272 subsequent medical complications such as pre-eclampsia or gestational

273 diabetes and those that delivered pre or post-term. We observed that in the

274 subgroup presenting with a normal birthweight at term, there was a significant

275 positive correlation between birthweight and the basal plate surface area

$276(p<0.001)$ and PV $(p<0.01)$. In this subgroup, we also found that the MS level

277 of $\beta$ hCG at $11-13^{+6}$ weeks was significantly higher $(\mathrm{p}<0.05)$ in women with a

$278 \mathrm{BMI} \geq 25$ than in women with a $\mathrm{BMI}<25$. First-trimester $3 \mathrm{D}$ placental volume

279 is closely associated with fetal and placental size [18] and hCG synthesis is

280 related to trophoblast proliferation and promotes development and growth of

281 the spiral arteries [29]. These findings highlight the pivotal interaction between

282 normal placentation, placental biosynthesis functions and normal fetal size,

283 and confirms that maternal BMI is a confounding factor that needs to be

284 adjusted for the first-trimester biochemical screening of trisomy 21.

In the LGA subgroup, we found that the MS PAPP-A at $11-13^{+6}$ weeks of gestation was significantly higher $(p<0.05)$ than in normal controls and was positively correlated with birthweight at term. PAPP-A is mainly produced by

288 the villous trophoblast and during pregnancy its synthesis is up-regulated by

289 progesterone, which promotes the adhesion and proliferation potential of

290 trophoblastic cells [30]. It is also a key regulator of insulin-like growth factor

291 bioavailability essential for normal fetal development. This can explain why at 
$29211-13^{+6}$ weeks of gestation, low MS levels of PAPP-A have been associated

293 with a higher risk of pre-eclampsia and poor fetal growth during the second

294 half of pregnancy [8-11,25,31]. Conversely, high MS PAPP-A levels as in the

295 present study have been associated with increased fetal size [21,32].

296 However, the sensitivity of MS PAPP-A at $11-13^{+6}$ weeks of gestation is too

297 low to be solely used as a screening method for the prediction of abnormal

298 birth weight and perinatal complications [8,9,11].

We found no difference in the ultrasound and biomarker parameters in the present study between SGA and normal birthweight controls. It is possible

301 that our small sample size in the SGA group could explain the lack of

302 relationship between the parameters and fetal size in the SGA group. The

303 conflicting results from studies using first and second trimester 3D placental

304 volume measurements to predict fetal size in the third trimester $[18,33]$ are

305 probably because of the difficulties with manual placental segmentation. In the

306 future innovative automatic segmentation approaches for 3D ultrasound may

307 be find placental measurements to be clinically applicable [34]. Variation in

308 placental volume is greater in the first trimester before 10 weeks i.e. before

309 the definitive placenta is fully formed [35], with a fundal or low placental

310 position being more difficult to assess than the fully anterior or posterior

311 position [33]. We have previously found that in pregnancies with a normal

312 outcome, there is no significant relationship between placental shape at 11-

$31313^{+6}$ weeks of gestation and birthweight or centile, suggesting that the

314 placental shape is not completely determined during the early phases of

315 placentation and is not directly related to fetal size during the first half of 316 pregnancy $[24,25]$. 
A distinct relationship exists between the uterine spiral arteries and the

318 fetal or placental lobules and the finding of a smaller basal plate surface area

319 at $11-13^{+6}$ weeks of gestation in pregnancies subsequently complicated by

320 pre-eclampsia [24]. This also suggests that ultrasound measurements of the

321 basal surface area reflects placentation indirectly and that this parameter is

322 less likely to be affected by the physiological morphological changes in the

323 placental structure between 8 and 12 weeks of gestation than placental

324 volume evaluated with 3D ultrasound.

\section{Conclusion}

326 In uncomplicated singleton pregnancies with a normal birthweight, 2D

327 measurements of placentation are related with fetal size but are not related to

328 subsequent excessive or slow fetal growth. LGA at birth is associated with

329 increased MS PAPP-A at 11-14 weeks of gestation supporting the association

330 between PAPP-A synthesis and early placental growth and development. The

331 clinical use of routine first trimester placental measurements in uncomplicated

332 pregnancies remains to be evaluated by large prospective cohorts. 
339 Acknowledgments: We acknowledge the major contribution to this study of

340 Dr Shanthi Muttukrishna, who sadly passed away before manuscript

341 submission. We thank Catherine Rogers and Dr Sangeeta Suri for patient

342 recruitment, data collection and follow up. We thank Paul Bassett for

343 statistical support. ALD has support at UCL/UCLH from the Department of

344 Health's NIHR Biomedical Research Centres funding scheme.

345 


\section{References}

1. Jauniaux E, Hempstock J, Greenwold N, Burton GJ. Trophoblastic oxidative stress in relation to temporal and regional differences in maternal placental blood flow in normal and abnormal early pregnancy. Am J Pathol 2003;162:115-125.

2. Burton GJ, Jauniaux $E$ and Charnock-Jones DS The influence of the intrauterine environment on human placental development. International Journal of Developmental Biology. 2010;54:303-12.

4. Burton GJ, Sibley CP, Jauniaux E. Placental Anatomy and Physiology.

3. Jauniaux E, Poston L, Burton GJ. Placental-related diseases of pregnancy: Involvement of oxidative stress and implications in human evolution. Hum Reprod Update. 2006;12:747-55.

5. Jaddoe VW, de Jonge LL, Hofman A, Franco OH, Steegers EA, Gaillard R. First trimester fetal growth restriction and cardiovascular risk factors in school age children: population based cohort study. BMJ. 2014;348:g14.

6. Burkhardt T, Schäffer L, Schneider C, Zimmermann R, Kurmanavicius J. Reference values for the weight of freshly delivered term placentas and for placental weight-birth weight ratios. Eur J Obstet Gynecol Reprod Biol. 2006;128:248-52.

7. Forbes K, Westwood M. Maternal growth factor regulation of human placental development and fetal growth. J Endocrinol. 2010;207:1-16.

8. Giudice I, Benintende G, Di Nicolò AM, et al. Correlation of neonatal weight with maternal serum levels of pregnancy-associated plasma protein-A during the first trimester of pregnancy: a retrospective study. $J$ Perinat Med. 2014. Leung TY, Sahota DS, Chan LW, Law LW, Fung TY, Leung TN, Lau TK. Prediction of birth weight by fetal crown-rump 
length and maternal serum levels of pregnancy-associated plasma protein-A in the first trimester. Ultrasound Obstet Gynecol. 2008;31:104.

9. Goetzinger KR, Singla A, Gerkowicz S, Dicke JM, Gray DL, Odibo AO. The efficiency of first-trimester serum analytes and maternal characteristics in predicting fetal growth disorders. Am J Obstet Gynecol. 2009;201:412.e1-6.

10. D'Antonio F, Rijo C, Thilaganathan B, Akolekar R, Khalil A, Papageourgiou A, Bhide A. Association between first-trimester maternal serum pregnancy-associated plasma protein-A and obstetric complications. Prenat Diagn. 2013;33(9):839-47.

11. Nanda S, Akolekar R, Sodre D, Vaikousi E, Nicolaides KH. Maternal serum adiponectin at 11-13 weeks of gestation in pregnancies delivering small for gestation neonates. Fetal Diagn Ther. 2011;29:2749.

12. Nanda S, Akolekar R, Sarquis R, Mosconi AP, Nicolaides KH. Maternal serum adiponectin at 11 to 13 weeks of gestation in the prediction of macrosomia. Prenat Diagn. 2011;31:479-83.

13. Jauniaux E, Jurkovic D, Campbell S, Hustin J. Doppler ultrasound study of the developing placental circulations: Correlation with anatomic findings. Am J Obstet Gynecol 1992;166:585-7.

14. Jauniaux E, Ramsay B, Campbell S. Ultrasonographic investigation of placental morphology and size during the second trimester of pregnancy. Am J Obstet Gynecol 1994;170:130-7.

15. Proctor LK, Toal M, Keating S, et al. Placental size and the prediction of severe early-onset intrauterine growth restriction in women with low pregnancy-associated plasma protein-A. Ultrasound Obstet Gynecol. 2009;34:274-82. 
16. Collins SL, Stevenson GN, Noble JA, Impey L. Rapid calculation of standardized placental volume at 11 to 13 weeks and the prediction of small for gestational age babies. Ultrasound Med Biol. 2013;39:253-60.

17. Effendi M, Demers S, Giguère $Y$, et al. Association between firsttrimester placental volume and birth weight. Placenta.2014;35:99-102.

18. Orzechowski K, Thomas D, McNamara CJ, Miller RC. Volumetric assessment of longitudinal placental growth. Obstet Gynecol. 2014;123:164S.

19. de Almeida Pimenta EJ, Silva de Paula CF, Duarte Bonini Campos JA, et al. Three-dimensional sonographic assessment of placental volume and vascularization in pregnancies complicated by hypertensive disorders. J Ultrasound Med. 2014;33:483-91.

20. Plasencia W, Akolekar R, Dagklis T, Veduta A, Nicolaides KH. Placental volume at 11-13 weeks' gestation in the prediction of birth weight percentile. Fetal Diagn Ther. 2011;30:23-8.

21. Schwartz N, Sammel MD, Leite R, Parry S. First-trimester placental ultrasound and maternal serum markers as predictors of small-forgestational-age infants. Am J Obstet Gynecol. 2014. pii: S00029378(14)00206-3.

22. Hata T, Tanaka H, Noguchi J, Hata K. Three-dimensional ultrasound evaluation of the placenta. Placenta.2011:32:105-15.

23. Olsen IE, Groveman SA, Lawson ML, Clark RH, Zemel BS. New intrauterine growth curves based on United States data. Pediatrics. 2010;125:e214-24.

24. Suri S, Muttukrishna S, Jauniaux E. 2D-ultrasound and endocrinologic evaluation of placentation in early pregnancy and its relationship to fetal birthweight in normal pregnancies and pre-eclampsia. Placenta. 2013;34:745-50. 
25. Jauniaux E, Suri S, Muttukrishna S. Evaluation of the impact of maternal smoking on ultrasound and endocrinological markers of first trimester placentation Early Hum Dev. 2013;89:777-80.

26. Hafner E, Metzenbauer M, Höfinger D, et al. Comparison between three-dimensional placental volume at 12 weeks and uterine artery impedance/notching at 22 weeks in screening for pregnancy-induced hypertension, pre-eclampsia and fetal growth restriction in a low-risk population. Ultrasound Obstet Gynecol 2006;27:652-7.

27. Toal M, Chan C, Fallah S, et al. Usefulness of a placental profile in high-risk pregnancies. Am J Obstet Gynecol 2007;196:363 e1-7.

28. Viero S, Chaddha V, Alkazaleh F, et al. Prognostic value of placental ultrasound in pregnancies complicated by absent end-diastolic flow velocity in the umbilical arteries. Placenta 2004;25:735-41.

29. Zygmunt M, Herr F, Keller-Schoenwetter S, et al. Characterization of human chorionic gonadotropin as a novel angiogenic factor. J Clin Endocrinol Metab. 2002;87:5290-6.

30. Wang J, Liu S, Qin HM, Zhao Y, Wang XQ, Yan Q. Pregnancyassociated plasma protein A up-regulated by progesterone promotes adhesion and proliferation of trophoblastic cells. Int J Clin Exp Pathol. 2014;7:1427-37.

31. Kalousová M, Muravská A, Zima T. Pregnancy-associated plasma protein A (PAPP-A) and preeclampsia. Adv Clin Chem. 2014;63:169209.

32. Poon LC, Karagiannis G, Stratieva V, Syngelaki A, Nicolaides KH. First-trimester prediction of macrosomia. Fetal Diagn Ther. 2011;29:139-47.

33. Hoopmann M, Schermuly S, Abele H, Zubke W, Kagan KO. First trimester pregnancy volumes and subsequent small for gestational age fetuses. Arch Gynecol Obstet. 2014;290:41-6. 
34. Stevenson GN, Collins SL, Ding J, Impey L, Noble AD. 3-D Ultrasound Segmentation of the Placenta Using the Random Walker Algorithm: Reliability and Agreement, Ultrasound Med. Biol. Sep. 2015 in press. 35. Salafia CM, Yampolsky M, Shlakhter A, Mandel DH, Schwartz N. Variety in placental shape: when does it originate? Placenta. 2012;33:164-70.

467 
Table 1. Comparison ( $\mathrm{W}$ test) of the birthweight, crown rump length $(\mathrm{CRL})$, ultrasound and endocrinological data in women with a booking BMI $<25(n=193)$ with those with a $B M I \geq 25(n=66)$. BMI was measured at $8-10$ weeks of gestation

\begin{tabular}{lccccc} 
Variables & \multicolumn{2}{c}{ BMI < 25 } & \multicolumn{2}{c}{ BMI $\geq 25$} \\
& Median & LQ;UQ & Median LQ;UQ & p \\
& & & & & \\
\hline CRL $(\mathrm{mm})$ & 63 & $56 ; 70$ & 62 & $57 ; 70$ & 0.80 \\
Birthweight $(\mathrm{g})$ & 3475 & $3226 ; 3689$ & 3454 & $3251 ; 3622$ & 0.89 \\
Placental thickness $\left(\mathrm{mm}^{2}\right)$ & 18.1 & $15.6 ; 21.6$ & 18.4 & $16.1 ; 21.8$ & 0.81 \\
Basal plate surface $\left(\mathrm{mm}^{2}\right)$ & 442 & $319 ; 531$ & 398 & $313 ; 517$ & 0.23 \\
Placental volume $\left(\mathrm{mm}^{3}\right)$ & 67.2 & $45.6 ; 94.7$ & 61.9 & $47.7 ; 77.8$ & 0.35 \\
fßhCG (MoM) & 0.98 & $0.69 ; 1.42$ & 1.20 & $0.76 ; 1.84$ & $<0.05$ \\
Inhibin A (MoM) & 0.95 & $0.74 ; 1.29$ & 1.10 & $0.80 ; 1.27$ & 0.23 \\
PAPP-A (MoM) & 1.08 & $0.74 ; 1.51$ & 0.95 & $0.70 ; 1.30$ & 0.19
\end{tabular}

Data are presented as median and lower quartile (LQ); upper quartile (UQ). 
Table 2. Comparison of the median for the ultrasound and endocrinological measurements of placental development in LGA neonates (birthweight $>90^{\text {th }}$ centile, $n=$ 30 ) and controls (birthweight $10^{\text {th }}-90^{\text {th }}$ centile, $n=90$ ).

Variables

LGA

Controls

Median LQ;UQ

Median LQ;UQ

$\mathrm{p}$

$\begin{array}{lrlcll}\text { Placental thickness }(\mathrm{mm}) & 18.2 & 15.2 ; 21.7 & 17.9 & 15.3 ; 22.0 & 0.91 \\ \text { Basal plate surface }\left(\mathrm{mm}^{2}\right) & 446 & 366 ; 522 & 453 & 346 ; 556 & 0.36 \\ \text { Placental volume }\left(\mathrm{mm}^{3}\right) & 63.4 & 47.7 ; 87.1 & 68.4 & 49.9 ; 87.5 & 0.71 \\ \text { fßhCG (MoM) } & 1.13 & 0.69 ; 1.59 & 1.09 & 0.72 ; 1.46 & 0.78 \\ \text { Inhibin A (MoM) } & 0.88 & 0.67 ; 1.18 & 1.02 & 0.78 ; 1.26 & 0.17 \\ \text { PAPP-A (MoM) } & 1.27 & 0.98 ; 1.73 & 1.03 & 0.74 ; 1.46 & <0.05\end{array}$

Data are presented as median and lower quartile (LQ); upper quartile (UQ). 
Table 3. Comparison of the median for the ultrasound and endocrinological measurements of placental development in SGA neonates (birthweight $\left\langle 10^{\text {th }}\right.$ centile, $n=$ 17) and controls (birthweight $10^{\text {th }}-90^{\text {th }}$ centile, $n=56$ ).

Variables

SGA

Controls

Median LQ;UQ

Median LQ;UQ

$\mathrm{p}$

$\begin{array}{lclcll}\text { Placental thickness }(\mathrm{mm}) & 17.5 & 14.7 ; 19.3 & 18.5 & 17.5 ; 21.5 & 0.11 \\ \text { Basal plate surface }\left(\mathrm{mm}^{2}\right) & 458 & 368 ; 586 & 443 & 318 ; 519 & 0.91 \\ \text { Placental volume }\left(\mathrm{mm}^{3}\right) & 64.5 & 52.7 ; 82.4 & 67.6 & 47.1 ; 88.6 & 0.27 \\ \text { fßhCG (MoM) } & 1.09 & 0.91 ; 1.58 & 1.11 & 0.75 ; 1.58 & 0.87 \\ \text { Inhibin A (MoM) } & 0.98 & 0.80 ; 1.08 & 1.09 & 0.78 ; 1.37 & 0.45 \\ \text { PAPP-A (MoM) } & 1.15 & 0.91 ; 1.26 & 1.08 & 0.74 ; 1.49 & 0.91\end{array}$

Data are presented as median and lower quartile (LQ); upper quartile (UQ).

468
469
470
471
472
473
474
475
476
477
478
479
480
481
482




\section{Figure 1 Legend}

Scatter plot illustrating the relationship between first trimester maternal serum

PAPP-A Multiple of the Median (MoM) and birthweight in the Large for

Gestational Age group. The regression line is indicated in blue, the red and

the purple lines indicate the confidence intervals.

\section{Supplementary Figure 1 Legend}

Scatter plot illustrating the relationship between first trimester placental

surface area and birthweight for the whole cohort. The regression line is

indicated in blue, the red and the purple lines indicate the confidence

intervals.

\section{Supplementary Figure 2 Legend}

Scatter plot illustrating the relationship between first trimester placental 2D

volume and birthweight for the whole cohort. The regression line is indicated

in blue, the red and the purple lines indicate the confidence intervals. 
Click here to download Figure: Figure 1 Scatter diagram.pdf

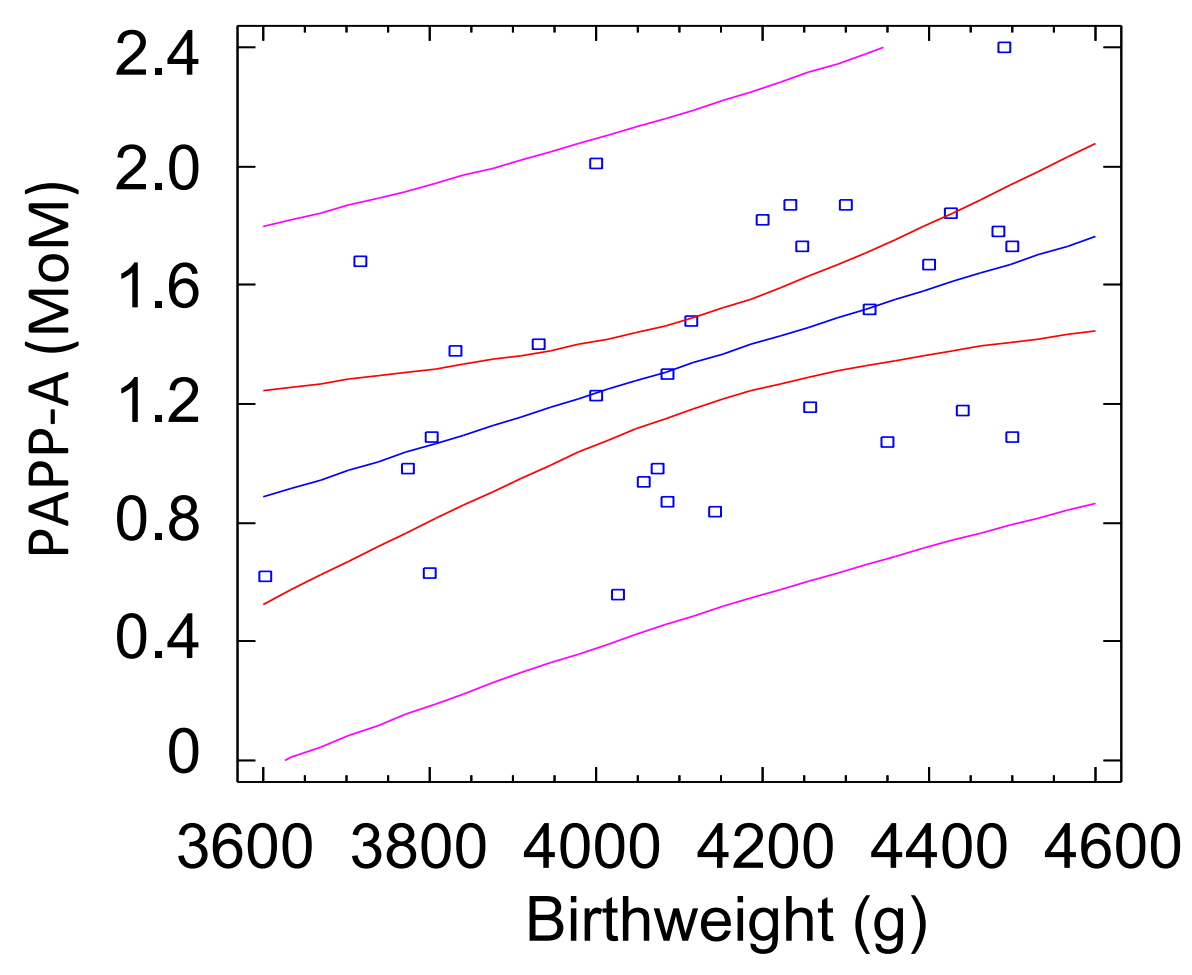


Supplementary File

Click here to download Supplementary File: Supplementary Figure 1 BW and Surface Area Scatter diagram.pdf .

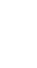

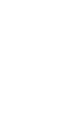



Supplementary File
Click here to downlo Supplementary File
Click here to download Supplementary File: Supplemental Figure 2 BW and volume Scatter diagram.pdf

\author{
Tupplemental Figure 2 BW and volume Scatter diagram.pdf
}

(1) $\sqrt{2}$ (1) (n) click here to download Supp 